\title{
Modeling the Complete Catalytic Cycle of Aspartoacylase
}

\section{Supporting Information}

\author{
Ekaterina D. Kots, ${ }^{\dagger+}$ Maria G. Khrenova, ${ }^{\dagger}$ Sofya V. Lushchekina, ${ }^{,}$

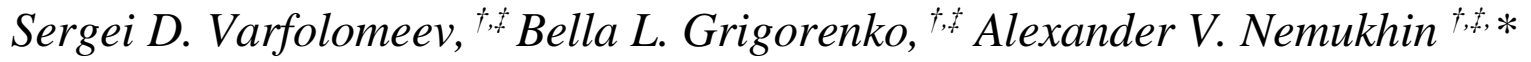 \\ ${ }^{\dagger}$ Chemistry Department, M.V. Lomonosov Moscow State University, Leninskie Gory 1/3, Moscow, \\ 119991, Russian Federation \\ ${ }^{*}$ N.M. Emanuel Institute of Biochemical Physics, Russian Academy of Sciences, Kosygina 4, \\ Moscow, 119334, Russian Federation \\ * Corresponding author: Prof. Alexander Nemukhin, Chemistry Department, M.V. Lomonosov \\ Moscow State University, Leninskie Gory 1/3, Moscow, 119991, Russian Federation \\ Phone: +7-495-939-10-96 \\ E-mail: anemukhin@yahoo.com, anem@lcc.chem.msu.ru
}

Section S1. QM/MM calculations with an extended QM region...................... P.S2

Section S2. Umbrella sampling Hamiltonian replica-exchange molecular

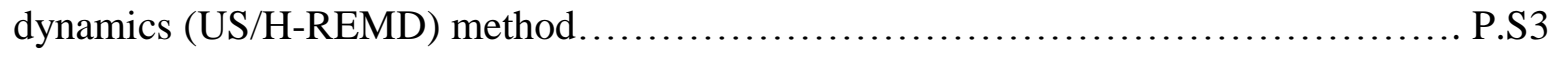

Section S3. Characterization of the structures of the ES complex and the

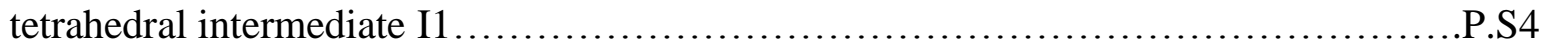

Section S4. View of the transport channels for substrate binding and product release...P.S7 
Section S1. QM/MM calculations with an extended QM region.

Scheme $\mathrm{S} 1$ shows the molecular groups assigned to the $\mathrm{QM}$ region in $\mathrm{QM} / \mathrm{MM}$ calculations. Table S1 compares the relative energies of the stationary points computed with the basic QM/MM model (Scheme 2 of the main text) and with the extended QM region in QM/MM. The density functional theory with the PBE0 functional and 6-31G** basis set was used in the QM part, the AMBER force field parameters were employed in MM.

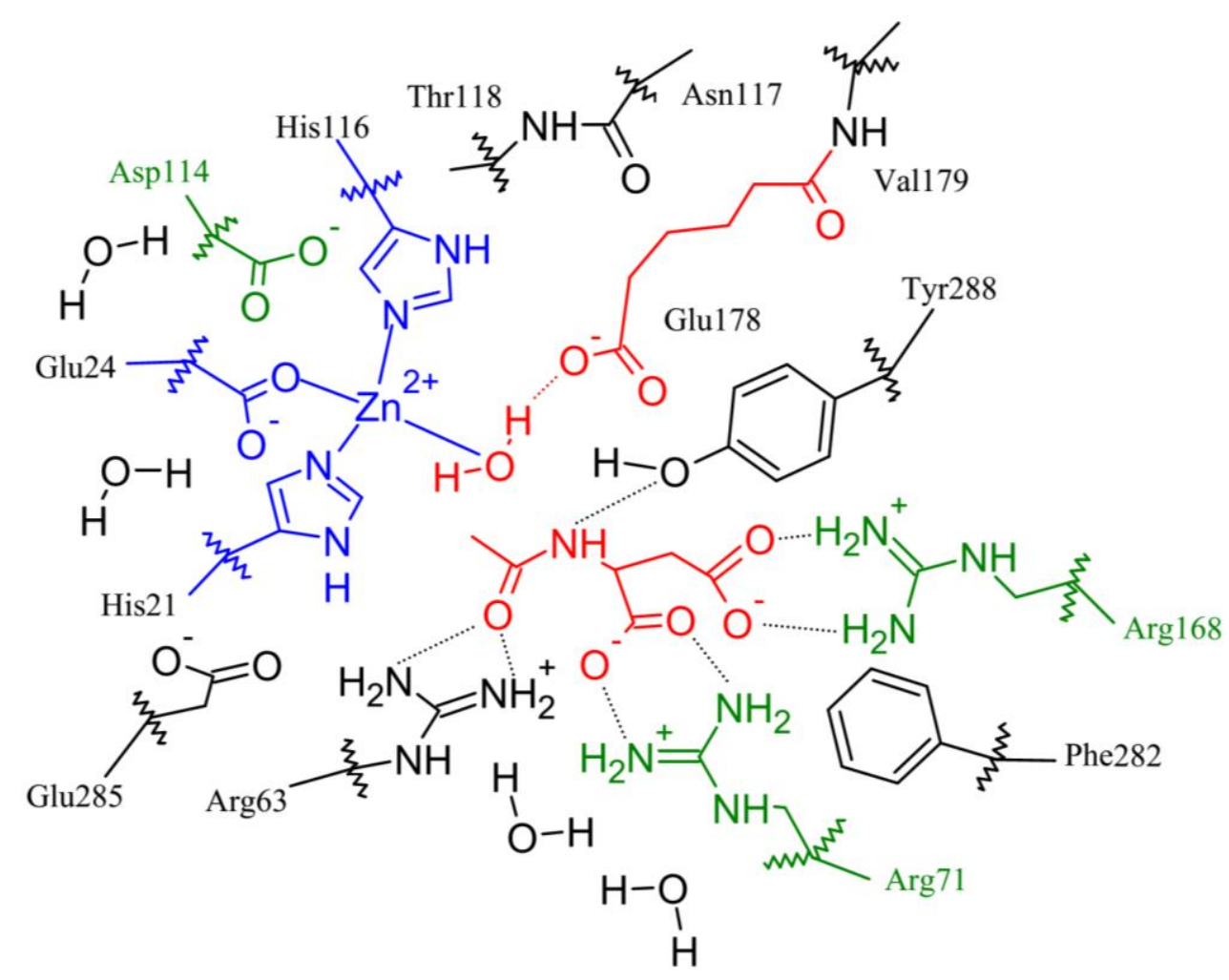

Scheme S1. The molecular groups assigned to the QM region. The immediate participants of the chemical transformations are shown in red, the coordination shell of zinc is colored blue. The groups colored green have not been included to the model described in the main text (Scheme 2). 
Table S1. Relative energies $(\mathrm{kcal} / \mathrm{mol})$ of the critical stationary points on the potential energy surfaces computed by the QM/MM method with the different QM regions.

\begin{tabular}{|l|c|c|}
\hline Stationary point & Basic QM region & Extended QM region \\
\hline ES & 0 & 0 \\
\hline TS1 & 12.5 & 10.1 \\
\hline I1 & 8.7 & 6.3 \\
\hline I2 & -2.7 & -2.5 \\
\hline I3 & 2.5 & 1.9 \\
\hline TS4 & 11.1 & 6.3 \\
\hline I4 & 1.6 & 0.2 \\
\hline I5 & 2.8 & -0.3 \\
\hline $\mathrm{EP}_{1} \mathrm{P}_{2}$ & 3.5 & -0.15 \\
\hline
\end{tabular}

Section S2. Umbrella sampling Hamiltonian replica-exchange molecular dynamics (US/H-REMD) method.

In the replica-exchange approach, several copies (replicas) of the molecular system are simulated simultaneously under slightly different Hamiltonians (H-REMD). At regular number of timesteps a swap between coordinates of replicas is accomplished. In the umbrella sampling (US) H-REMD, a biasing potential $V(d, i)$ is added to each Hamiltonian of $n$ created replicas:

$$
V(d, i)=\frac{1}{2} k\left(d-d_{i}\right)^{2}
$$

Here $i$ ranges from 0 to $n-1, k$ is a force constant, $d$ is a variable along which the potential of mean force is calculated and $d_{i}$ is a center of the biasing potential of the $i$-replica. Within the simulation, the energies of neighboring replicas are compared periodically and their biasing potential exchange with a specified transition probability. The resulting values of collective variable along the trajectory are further processed with the weighted histogram analysis method. 
Application of the H-REMD protocol for the case of substrate (NAA) delivery to the ASPA active site (see main text) is illustrated in Fig.S1.

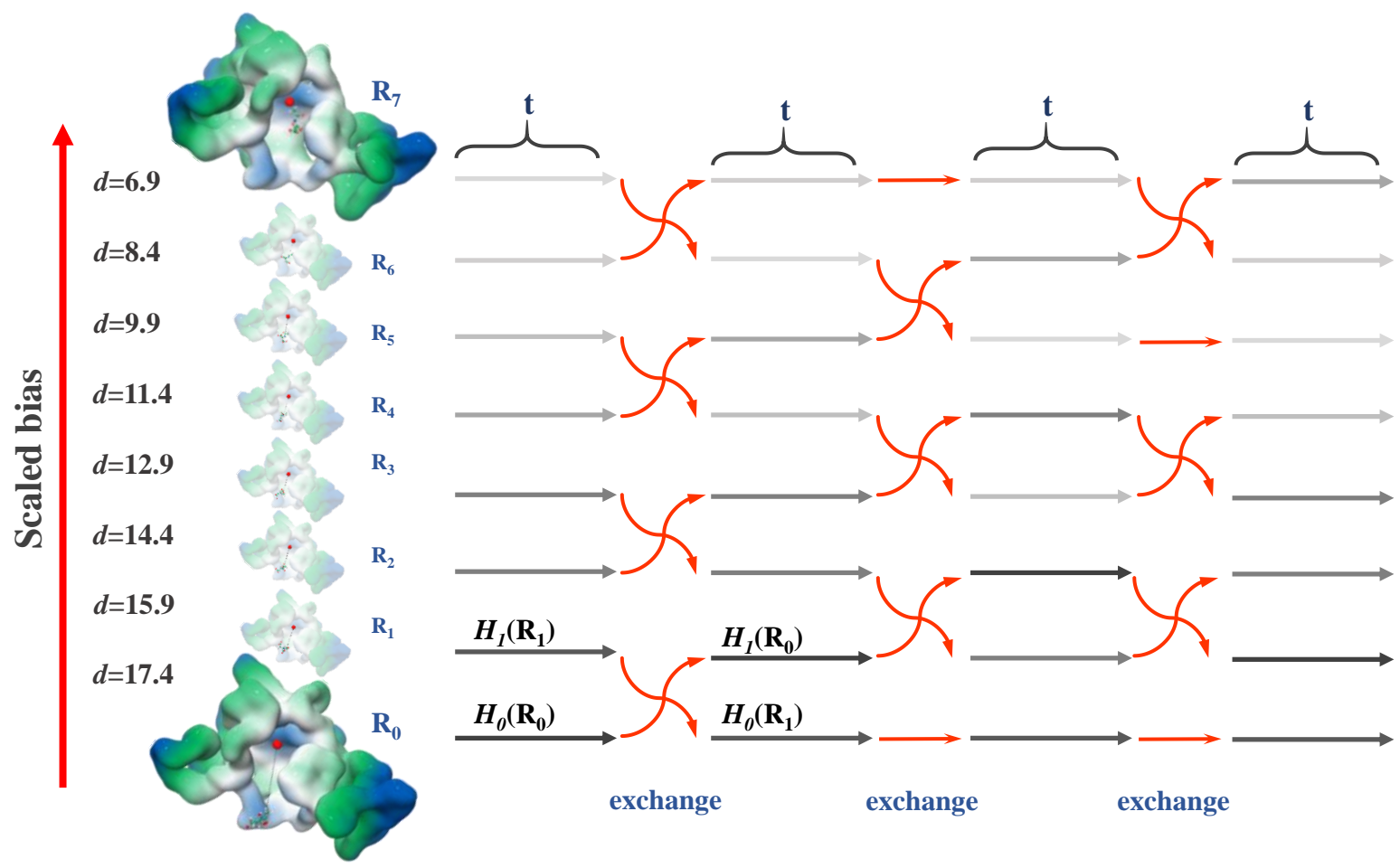

Figure S1. Illustration of the H-REMD protocol for the case of substrate binding to the active site.

Section S3. Characterization of the structures of the ES complex and the tetrahedral intermediate I1.

The computed equilibrium geometry configuration of the first reaction intermediate I1 of our model system corresponds to the crystal structure PDBid:2O4H for the complex of ASPA with Nphosphonomethyl-L-aspartate covalently bound to the protein. Fig. 2 of the main text demonstrates a superposition of the molecular groups at the active site of the computed and crystal structures. Table S1 compares explicitly the most important geometry parameters for I1.

Table S2 compares the key distances in the zinc coordination shell near the ES structure obtained by using different computational protocols. Fig.S2 illustrates the graph of the Ow(Wat)-Cs(NAA) distances near the ES structure along the MD-QM/MM trajectory. 
Table S1. Comparison of selected distances $(\AA)$ between heavy atoms in the crystal structure PDBid:2O4H and in the computed model system mimicking the first reaction intermediate I1.

\begin{tabular}{|c|c|c|}
\hline Distances & $\begin{array}{c}\text { Crystal structure } \\
\text { PDBid:2O4H }\end{array}$ & $\begin{array}{l}\text { Computed structure of } \\
\text { the intermediate I1 }\end{array}$ \\
\hline $\mathrm{Zn}-\mathrm{Ow}$ (NAA) & 2.43 & 2.61 \\
\hline $\mathrm{Zn}-\mathrm{Os}(\mathrm{NAA})$ & 2.55 & 1.93 \\
\hline $\mathrm{Zn}-\mathrm{N} \delta 1$ (His21) & 2.23 & 2.06 \\
\hline Zn - N $\delta 1$ (His116) & 2.29 & 2.02 \\
\hline $\mathrm{Zn}-\mathrm{O} \varepsilon 1$ (Glu24) & 2.15 & 2.05 \\
\hline $\mathrm{Zn}-\mathrm{O} 2$ (Glu24) & 2.62 & 2.58 \\
\hline Ow (NAA) - Oع2 (Glu178) & 2.93 & 2.66 \\
\hline Ns (NAA) - O $\varepsilon 1$ (Glu178) & 3.74 & 3.40 \\
\hline 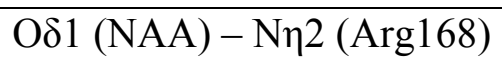 & 2.94 & 2.67 \\
\hline O $\delta 2$ (NAA) - N 1 (Arg168) & 3.26 & 2.70 \\
\hline 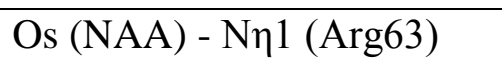 & 2.84 & 2.73 \\
\hline 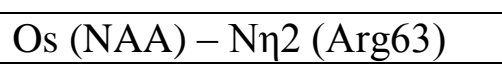 & 3.00 & 3.55 \\
\hline
\end{tabular}


Table S2. Comparison of the key distances in the zinc coordination shell $(\AA)$ near the ES structure obtained with the different computational protocols: the $\mathrm{QM}\left(\mathrm{PBE} 0 / 6-31 \mathrm{G}^{* *}\right) / \mathrm{MM}(\mathrm{AMBER})$ optimized distances for the basic and extended QM regions, the MD average values obtained with the $\mathrm{QM}(\mathrm{PBE} / \mathrm{DZVP}-\mathrm{PW}) / \mathrm{MM}(\mathrm{CHARMM})$ potentials and the energy minimized distances using the QM(PBE/DZVP-PW)/MM(CHARMM) calculations.

\begin{tabular}{|l|c|c|c|c|}
\hline Distances & $\begin{array}{c}\text { Basic QM } \\
\text { region }\end{array}$ & $\begin{array}{c}\text { Extended } \\
\text { QM region }\end{array}$ & $\begin{array}{c}\text { Average } \\
\text { MD QM/MM }\end{array}$ & $\begin{array}{c}\text { Optimized } \\
\text { MD QM/MM }\end{array}$ \\
\hline $\mathrm{Zn}-\mathrm{O} \varepsilon 1$ Glu24 & 2.18 & 2.18 & $2.09 \pm 0.11$ & 2.17 \\
\hline $\mathrm{Zn}-\mathrm{O} \varepsilon 2$ Glu24 & 2.28 & 2.26 & $2.57 \pm 0.27$ & 2.30 \\
\hline $\mathrm{Zn}-\mathrm{N} \delta$ His21 & 2.18 & 2.16 & $2.12 \pm 0.11$ & 2.19 \\
\hline $\mathrm{Zn}-\mathrm{N} \delta$ His116 & 2.06 & 2.04 & $2.06 \pm 0.08$ & 2.12 \\
\hline $\mathrm{Zn}-\mathrm{O}$ Wat & 2.04 & 2.02 & $2.05 \pm 0.08$ & 2.04 \\
\hline
\end{tabular}

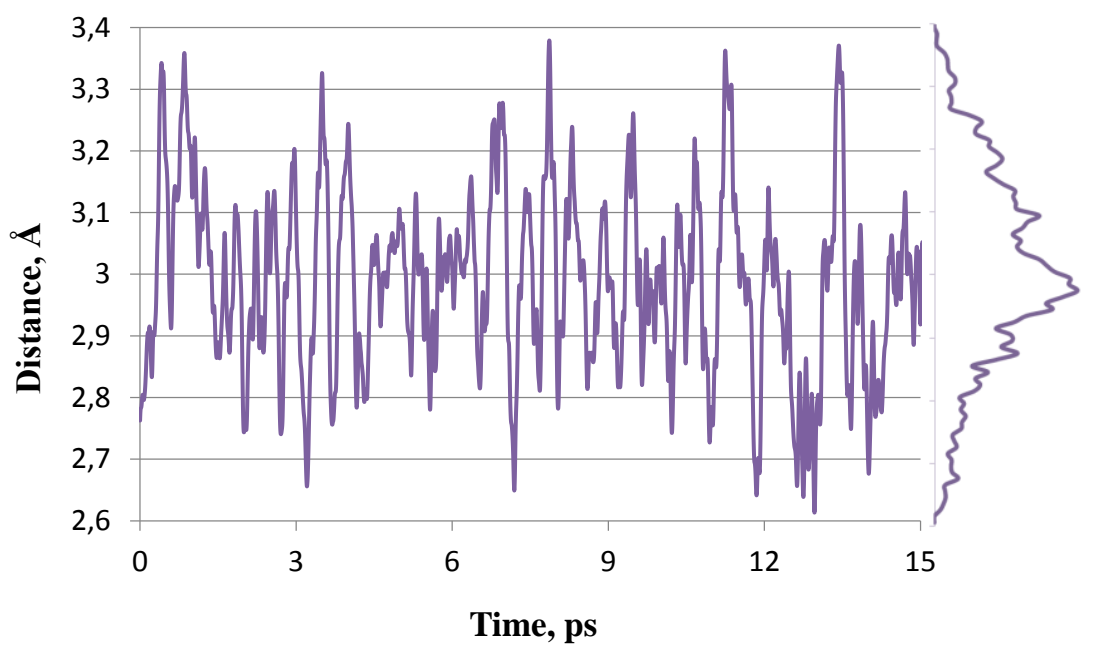

Figure S2. The graph of the Ow(Wat)-Cs(NAA) distances along the MD-QM/MM trajectory near the ES complex. The right panel shows the distribution of distances. 
Section S4. View of the transport channels for substrate binding and product release.

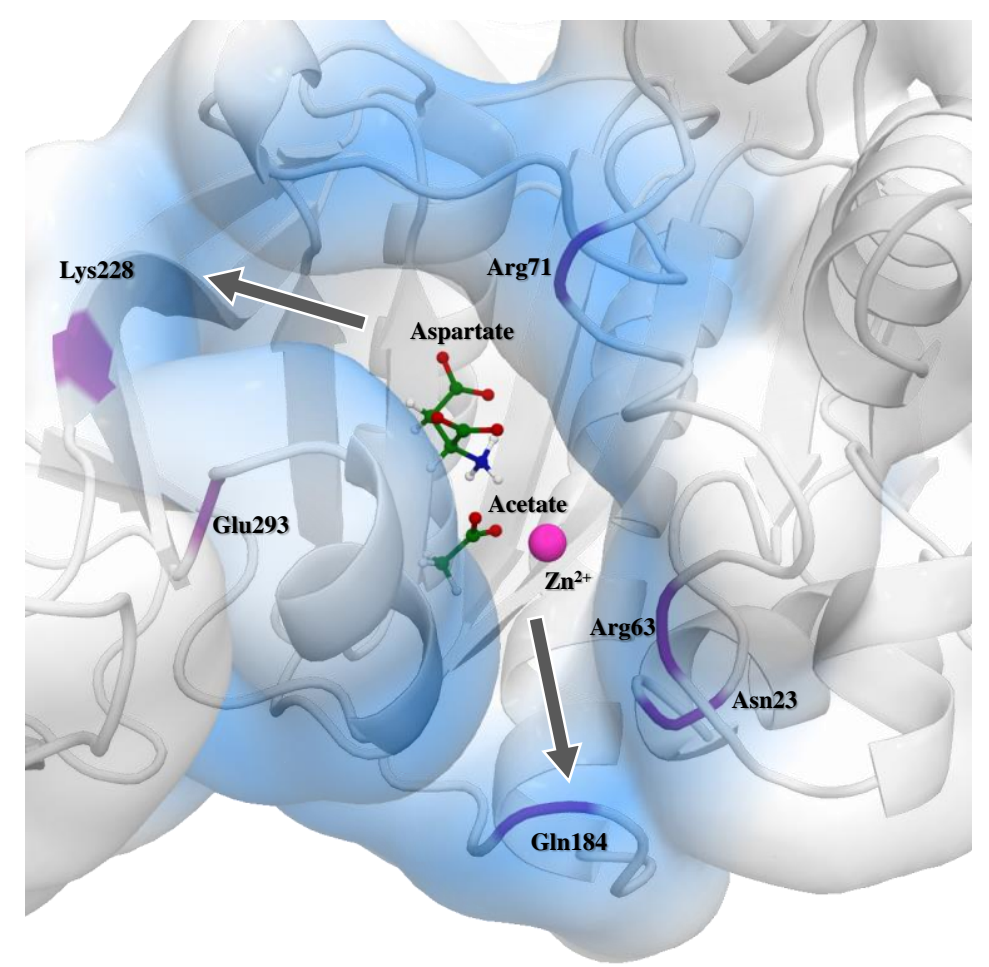

Figure S3. Transport channels in the structure of ASPA. Substrate delivery and exit of the aspartate occur through the channel gated by the side chains of Arg71, Lys228 and Glu293. Release of the acetate occurs through another channel gated by the side chains of Asn23, Arg63 and Gln184. 